\title{
Multiplets and Terms in Technetium Spectra
}

\author{
William F. Meggers
}

\begin{abstract}
Multiplets and terms of the quartet, sextet, and octet systems are reported for the first spectrum of Tc. The combinations of 20 terms composed of 63 atomic energy levels account for $200 \mathrm{Te}$ I lines and 66 percent of the total observed intensity summed for 1,300 lines.

The ground state of neutral Tc atoms is represented by $4 d^{5} 5 s^{2}{ }^{6} \mathrm{~S}_{213}$, and the first metastable state by $4 d^{6} 5 s^{6} \mathrm{D}_{43}$, only $2573 \mathrm{~cm}^{-1}$ higher. A Rydberg series of ${ }^{8 \mathrm{~S}}$ terms yields absolute term values from which a Ritz-corrected limit of $60070 \mathrm{~cm}^{-1}$, or ionization potential of 7.45 electron volts, is derived.

In the second spectrum of Te the principal S and $\mathrm{P}$ terms of the quintet and septet systems are identified and fixed relative to each other. Evidence is presented that $4 d^{6}{ }^{5} \mathrm{D}$ represents the normal state of $\mathrm{Te}^{+}$ions, but to confirm this further observations of the Te II spectrum are necessary.
\end{abstract}

\section{Introduction}

In 1922 Catalán [1] ${ }^{1}$ discovered, in the spectrum of manganese, that certain groups of lines having the same character are related by very exact numerical separations, and he suggested that the word "multiplet" be used to denote such groups. Since that time the search for multiplets in all atomic spectra has been the chief objective of spectrum analysis. This search, aided by the quantum theory, has resulted in present-day information concerning spectral terms, atomic structures, ionization potentials, and many other properties of atoms, of ions, and of atomic nuclei. The purpose of this paper is to report on information of this type derived from preliminary analyses of the first two spectra of technetium, the most recently discovered chemical homolog of manganese.

This chemical element was predicted in 1869 , by Mendeléeff who found that when the then-known chemical elements were arranged in order of increasing atomic weights, their maximum chemical valencies exhibited periodicities, with gaps suggesting two unknown homologs of manganese. In 1925 Noddack and Tacke [2] announced the discovery of both of these elements and proposed for them the names "masurium" and "rhenium." Rhenium soon became available in abundance, and in 1931 Meggers published a preliminary description of its arc spectrum, also reporting multiplets, atomic energy levels, and the ionization potential for Re I. The separation of masurium from natural minerals has never been repeated or confirmed, but the element in question has recently been produced artificially either by transmutation of molybdenum [3] or by fission of uranium [4]. This artificial element was named [5] technetium (symbol Tc). A preliminary description of the arc and spark spectra of Tc by Meggers and Scribner [6] constitutes the material in which the first multiplets and spectral terms of Tc were found.

The basic spectral data consist of measured wavelengths and estimated relative intensities of more than 2,200 radiations characteristic of Tc atoms and

\footnotetext{
1 Figures in brackets indicate the literature references at the end of this paper.
}

ions. These spectral lines are divided into two classes, one (Tc I) characteristic of neutral Tc atoms, and the other (Tc II) of singly ionized atoms. A considerable number of Tc lines were observed to be complex $(c)$ with hyperfine structure, and many were hazy $(h)$ in the spark spectrograms. Other criteria, such as reversibility, pressure displacement, temperature classification, and Zeeman effect, which are extremely helpful in the analyses of complex spectra, are still wholly lacking for Tc lines. In the absence of these aids it might appear presumptuous to attempt the analyses of Tc spectra, but fortunately these spectra are so well-observed and orthodox that the principal multiplets could be identified without difficulty. The first guiding principles in the search for Tc multiplets were the interval and intensity rules, assuming that $L S$-coupling controls the optical electrons. The second clues to Tc multiplets came from comparisons of analogous $\mathrm{Mn}$ and Te spectra as regards term intervals, term magnitudes, and electron configurations. Under these conditions a reliable list of lines, wavelengths, and intensities will yield atomic information, provided that the observed spectral region and range are right. This statement is illustrated by the atomic information derived from the analyses of Te I and Te II spectra.

\section{First Spectrum of Technetium, $\mathrm{T}_{\mathrm{I}}$}

\section{TC I Multiplets}

The observed data of the Tc I spectrum comprise about 1,300 lines with wavelengths ranging from 2324.57 to $8829.80 \mathrm{~A}$, and estimated intensities ranging from 1 to 500. Vacuum wave numbers corresponding to the measured wavelengths of Te I lines were obtained from Kayser's Tabelle der Schwingungszahlen [7]. Like other first spectra of metals, the Tc I spectrum is weak in the ultraviolet, strong in the short-wave visible and adjacent ultraviolet, and lacks lines of high intensity in the near infrared. My search for multiplets in this spectrum began with the identification of a group of three lines $\left(a^{6} \mathrm{~S}_{21 / 2}-z^{6} \mathrm{P}_{31 / 2}^{\circ}, 21 / 2,11 / 2\right)$ which are outstanding 
in the analogous Mn I $(4030.76,4033.07,4034.49 \mathrm{~A})$ and $\operatorname{Re} I(3451.88,3466.47,3464.72 \mathrm{~A})$ spectra.

Naively, I interpolated between Mn I and Re I, and looked for the analogous Tc I lines between 3700 and $3800 \mathrm{~A}$, but failed to find them there. The most intense Tc I lines have wavelengths 4297.06, 4262.26, $4238.19 \mathrm{~A}$. The wave number separations (189.9 and $133.2 \mathrm{~cm}^{-1}$ ) of these three lines are almost exactly proportional to 7 and 5, which characterize a good ${ }^{6} \mathrm{P}$ term. The same separations were next found among seven weak lines (4948 to 5139 A), but the significance of these was not recognized until recurring differences (302.1 and $449.7 \mathrm{~cm}^{-1}$ ) found between wave numbers of strong lines (3500 to $4200 \mathrm{~A}$ ) were seen to be identical with the cross differences of the seven weak lines. This led at once to the identification and fixation of the metastable five-level term, $a{ }^{6} \mathrm{D}$, and many multiplets that involve this term. Surplus $a^{6} \mathrm{D}$ separations among other Tc I lines were regarded as belonging to intersystem combinations, which gave the clue to the fixation and identification of a metastable four-level term $a^{4} \mathrm{D}$, and multiplets that involve this term.

Finally, a unique pair of visible Tc I lines (5924.57 and $6085.22 \mathrm{~A}$ ), exhibiting the widest hyperfine structure, were assumed to represent the transition $a^{6} \mathrm{~S}_{21 / 2}-z^{8} \mathrm{P}_{31 / 2,21 / 2}^{0}$.
This assumption was confirmed by the establishment of $e^{8} \mathrm{~S}$ and $e^{8} \mathrm{D}$, which give with $z^{8} \mathrm{P}^{\circ}$ the strongest lines in the octet system. The principal multiplets of the Tc I spectrum are displayed in tables 1,2 , and 3 , where the term symbols and values (relative to $a{ }^{6} \mathrm{~S}_{2 \frac{1}{2}}=0.0$ ) are shown at the top and left. Differences of these level values are entered in the body of the table where the levels intersect. These numbers are the actual wave numbers of all lines permitted by the selection rule $\Delta J=0, \pm 1$. The observed wavelengths and intensities (parentheses) appear directly above the wave numbers. The average difference between computed and observed wave numbers is $\pm 0.06 \mathrm{~cm}^{-1}$, which proves that the measured wavelengths of these Tc lines are rarely in error by more than $0.01 \mathrm{~A}$ in relative value. The total number of permitted lines in tables 1, 2, 3 is 240, whereas the number of observed lines is 200 . In a few cases the unobserved Tc I lines are obscured by Tc II, by impurity, or by strong copper lines, but in most cases the absence of predicted combinations is explained by the fact that they are either weak intersystem transitions or double-electron jumps. One line $4145.0 \mathrm{~A}$ is doubly classified; the calculated components are separated by $1 \mathrm{~cm}^{-1}$ but were not resolved because of unequal intensities.

TABLE 1. Multiplets in the Tc I spectrum

\begin{tabular}{|c|c|c|c|c|c|c|c|}
\hline & $\begin{array}{l}\text { a }{ }^{6} \mathrm{~S}_{21 / 2} \\
0.0\end{array}$ & $\begin{array}{l}a^{6}{ }^{6} \mathrm{D}_{41 / 2} \\
2572.9\end{array}$ & $\begin{array}{l}a^{6}{ }^{6} \mathrm{D}_{31 / 2} \\
3250.9\end{array}$ & $\begin{array}{l}a^{6}{ }^{6} \mathrm{D}_{21 / 2} \\
3700.6\end{array}$ & $\begin{array}{l}a^{6}{ }^{6} \mathrm{D}_{11 / 2} \\
4002.7\end{array}$ & $\begin{array}{l}a^{6}{ }^{6} \mathrm{D}_{01 / 2} \\
4178.8\end{array}$ & $\begin{array}{l}e^{6} \mathbf{S}_{21 / 2} \\
39599.0\end{array}$ \\
\hline $\begin{array}{l}z^{6} \mathrm{P}_{31 / 2}^{\circ} \\
23265.4 \\
z^{6} \mathrm{P}_{21 / 2}^{\circ} \\
23455.3 \\
z^{6} \mathrm{P}_{11 / 2}^{\circ} \\
23588.5 \\
z^{6} \mathrm{D}_{41 / 2}^{\circ} \\
27369.8 \\
z^{6} \mathrm{D}_{31 / 2}^{\circ} \\
27660.1 \\
z^{6} \mathrm{D}_{21 / 2}^{\circ} \\
27940.8 \\
z^{6} \mathrm{D}_{11 / 2}^{\circ} \\
28151.4 \\
z^{6} \mathrm{D}_{01 / 2}^{\circ} \\
28296.7 \\
z^{\circ} \mathrm{F}_{51 / 2}^{\circ} \\
30067.1 \\
z^{6} \mathrm{~F}_{41 / 2}^{\circ} \\
30133.1 \\
z^{6} \mathrm{~F}_{31 / 2}^{\circ} \\
30382.0 \\
z^{6} \mathrm{~F}_{21 / 2}^{\circ} \\
30528.8 \\
z^{6} \mathrm{~F}_{11 / 2}^{\circ} \\
30630.5 \\
z^{6} \mathrm{~F}_{01 / 2}^{\circ} \\
30689.1 \\
x^{\circ} \mathrm{P}_{31 / 2}^{\circ} \\
31414.0 \\
x^{6} \mathrm{P}_{21 / 2}^{\circ} \\
31406.7 \\
x^{6} \mathrm{P}_{11 / 2}^{\circ} \\
31503.8\end{array}$ & $\begin{array}{l} \\
3182.38(30) \\
31414.0 \\
3183.12(30) \\
31406.7 \\
3173.30(40) \\
31503.9\end{array}$ & $\begin{array}{l}3636.10(300) \\
27494.2 \\
3627.39(25) \\
27560.2 \\
3594.93(2) \\
27809.1\end{array}$ & $\begin{array}{l}4995.00(15) \\
20014.5 \\
4948.06(8) \\
20204.3 \\
\\
\\
4145.02(80) \\
24118.6 \\
4095.68(150) \\
24409.1 \\
4049.10(150) \\
24689.9 \\
\end{array}$ & $\begin{array}{l}5109.79(8) \\
19564.8 \\
5060.69(30) \\
19754.7 \\
5026.79(5) \\
19887.9 \\
\\
\\
4172.52(80) \\
23959.5 \\
4124.22(60) \\
24240.2 \\
4088.70(100) \\
24450.8\end{array}$ & $\begin{array}{l}3768.80(100) \\
26526.1 \\
3754.41(50) \\
26627.8 \\
3746.18(15) \\
26686.3 \\
\\
\\
3648.07(80) \\
27404.0 \\
3635.18(80) \\
27501.1\end{array}$ & $\begin{array}{l}4170.28(50) \\
23972.6 \\
4145.02(80) \\
24118.6\end{array}$ & $\begin{array}{l}6120.67(150) \\
16333.6 \\
6192.65(100) \\
16143.7 \\
6244.17(50) \\
16010.5\end{array}$ \\
\hline
\end{tabular}


TABle 1. Multiplets in the Te I spectrum-Continued

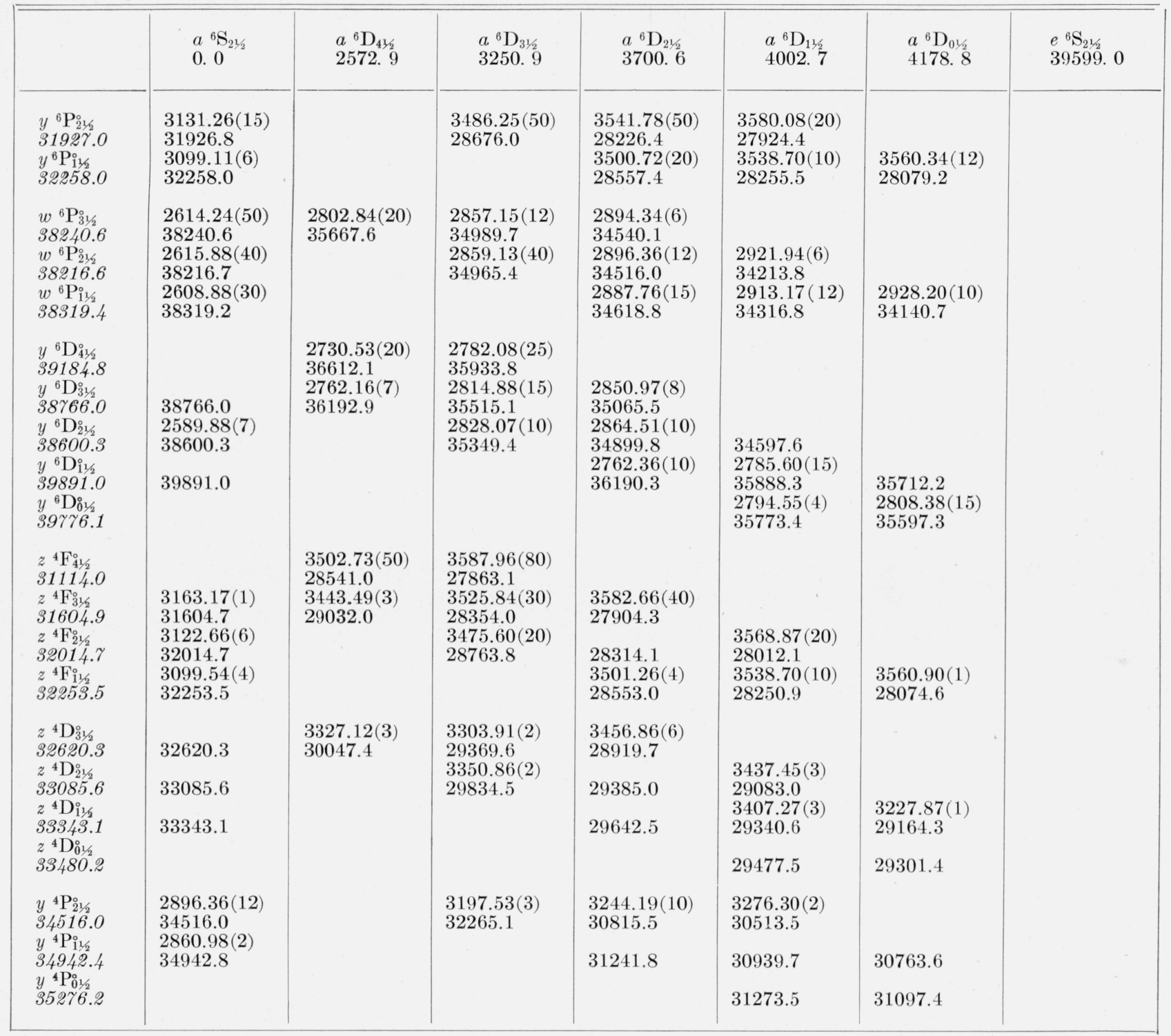


TABLE 2. Multiplets in the Tc I spectrum

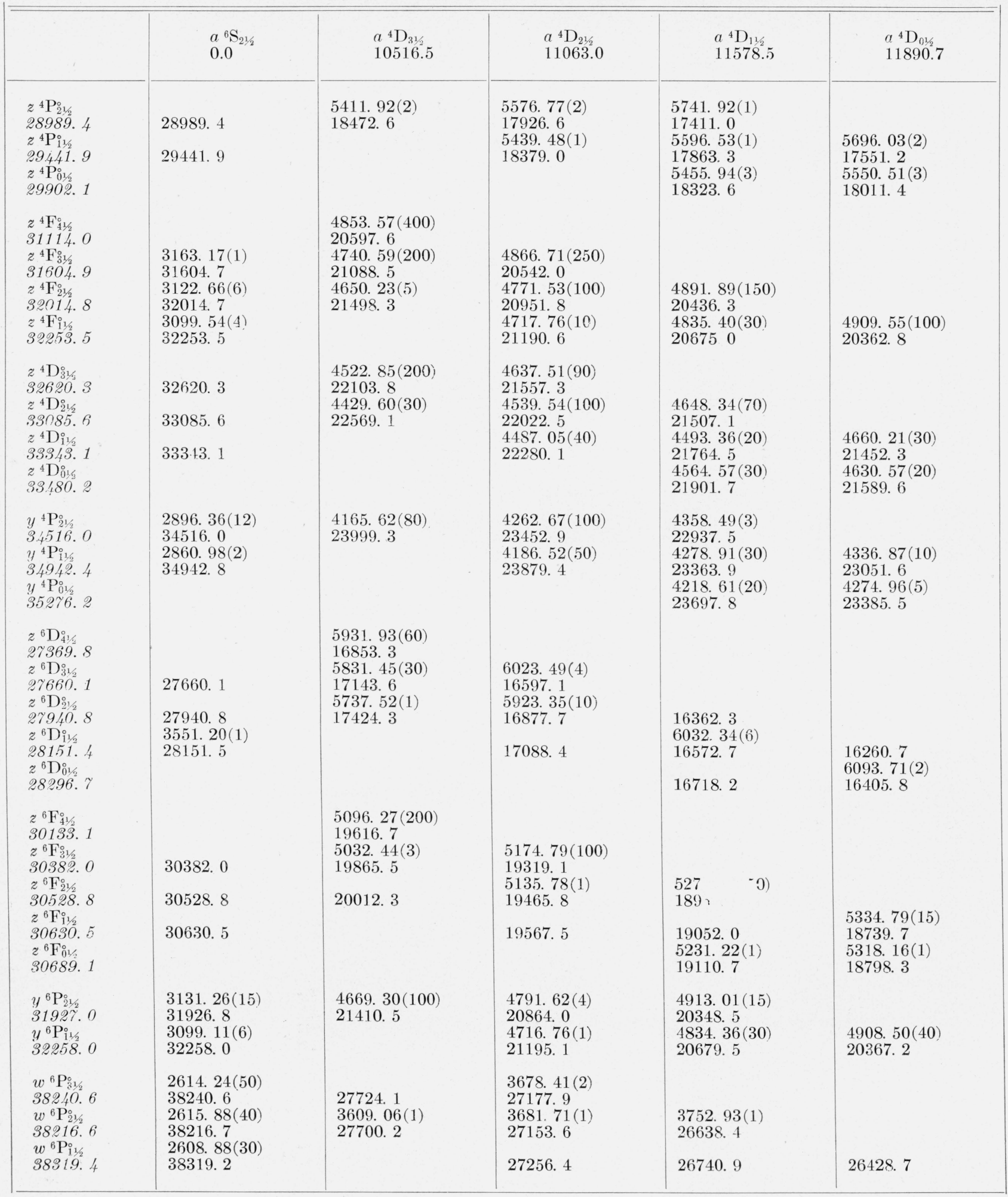


TaBle 3. Multiplets in the Te I spectrum

\begin{tabular}{|c|c|c|c|}
\hline & $\begin{array}{l}z^{8} P_{41 / 2}^{\circ} \\
17523.0\end{array}$ & $\begin{array}{l}z^{8} P_{31 / 2}^{\circ} \\
16874.5\end{array}$ & $\begin{array}{l}z^{8} P_{21 / 2}^{\circ} \\
16428.8\end{array}$ \\
\hline $\begin{array}{l}\text { a }{ }^{6} \mathrm{~S}_{21 / 2} \\
0.0\end{array}$ & & $\begin{array}{l}5924.57(100) \\
16874.5\end{array}$ & $\begin{array}{l}\text { 6085. } 22(100) \\
16428.7\end{array}$ \\
\hline $\begin{array}{l}a^{6} \mathrm{D}_{41 / 2} \\
2572.9\end{array}$ & $\begin{array}{l}\text { 6687. } 03(3) \\
14950.2\end{array}$ & $\begin{array}{l}\text { 6990. } 22(1) \\
14301.8\end{array}$ & \\
\hline $\begin{array}{l}a^{6}{ }^{6} \mathrm{D}_{31 / 2} \\
3250.9\end{array}$ & 14272. 1 & $\begin{array}{l}\text { 7338. } 18(3) \\
13623.6\end{array}$ & 13177. 9 \\
\hline $\begin{array}{l}a^{6} \mathrm{D}_{21 / 2} \\
3700.6\end{array}$ & & 13173. 9 & $\begin{array}{l}\text { 7854. } 35(1) \\
12728.3\end{array}$ \\
\hline $\begin{array}{l}a{ }^{6} \mathrm{D}_{11 / 2} \\
4002.7\end{array}$ & & & $\begin{array}{l}8045.30(2) \\
12426.2\end{array}$ \\
\hline $\begin{array}{l}e^{8} \mathrm{~S}_{31 / 2} \\
37612.5\end{array}$ & $\begin{array}{l}4976.34(200) \\
20089.5\end{array}$ & $\begin{array}{l}\text { 4820. } 75(150) \\
20737.9\end{array}$ & $\begin{array}{l}\text { 4719. } 28(100) \\
21183.8\end{array}$ \\
\hline $\begin{array}{l}e^{8} \mathrm{D}_{51 / 2} \\
44365.3\end{array}$ & $\begin{array}{l}3724.41(50) \\
26842.3\end{array}$ & & \\
\hline $\begin{array}{l}e^{8} \mathrm{D}_{41 / 2} \\
44352.5\end{array}$ & $\begin{array}{l}3726.19(10) \\
26829.5\end{array}$ & $\begin{array}{l}\text { 3638. 24(40) } \\
27478.0\end{array}$ & \\
\hline $\begin{array}{l}e^{8} \mathrm{D}_{31 / 2} \\
44343.9\end{array}$ & $\begin{array}{l}\text { 3727. } 37(3) \\
26821.0\end{array}$ & $\begin{array}{l}3639.41(30) \\
27469.2\end{array}$ & $\begin{array}{l}\text { 3581. } 27(20) \\
27915.1\end{array}$ \\
\hline $\begin{array}{l}e^{8} \mathrm{D}_{21 / 2} \\
44337.5\end{array}$ & & $\begin{array}{l}\text { 3640. 23(10) } \\
27463.0\end{array}$ & $\begin{array}{l}3582.10(15) \\
27908.7\end{array}$ \\
\hline $\begin{array}{l}e^{8} \mathrm{D}_{11 / 2} \\
44333.1\end{array}$ & & & $\begin{array}{l}3582.66(40) \\
27904.3\end{array}$ \\
\hline $\begin{array}{l}f^{8} \mathbf{S}_{31 / 2} \\
49573.3\end{array}$ & $\begin{array}{l}3119.18(3) \\
32050.4\end{array}$ & $\begin{array}{l}\text { 3057. } 34(2) \\
32698.7\end{array}$ & $\begin{array}{l}3016.31(1) \\
33143.5\end{array}$ \\
\hline
\end{tabular}

Although the 200 classified lines presented here comprise only 15 percent of the total number of Te I lines, they account for 66 percent of the total intensity, summed for 1,300 observed lines.

\section{Tc r Spectral Terms}

The Tc I multiplets in tables $1,2,3$ result from combinations of 20 spectral terms embracing 63 atomic energy levels that are arrayed in table 4 alongside the analogous terms and levels belonging to the Mn I spectrum [8]. The electron configurations in column 1 apply to $\mathrm{Mn} \mathrm{I}$; for Te I the principal quantum numbers must be increased by one unit. Naturally, the intervals, between levels of complex terms are larger in $\mathrm{Tc}$ than in $\mathrm{Mn}$, but the algebraic signs, with few exceptions, are the same. A plus sign means that the term is regular, minus means inverted. The Mn I terms are arranged in order of increasing magnitude of levels having the largest $J$ but the analogus Tc I terms depart from this order because the sixth $d$-type electron is more firmly bound in Tc than in Mn. Thus, in Mn I $3 d^{6} 4 s^{6} \mathrm{D}_{41 / 2}$ is $17052.3 \mathrm{~cm}^{-1}$ above the ground state $3 d^{5} 4 s^{2}{ }^{6} \mathrm{~S}_{21 / 2}$, whereas in Tc I $4 d^{6} 5 s^{6} \mathrm{D}_{4 \frac{1}{2}}$ is only $2572.9 \mathrm{~cm}^{-1}$ above zero. Similarly, the associated ${ }^{4} \mathrm{D}_{31 / 2}$ levels are, respectively, 23296.7 and $10516.5 \mathrm{~cm}^{-1}$ above zero. It appears that terms from configurations containing six $d$-electrons are displaced about 13,000 $\mathrm{cm}^{-1}$, whereas those from configurations with five $d$-electrons are displaced only about $2,000 \mathrm{~cm}^{-1}$. These displacements are shown in the last column of table 4; they served as a guide in assigning Te I terms to the electron configurations in the first column. After the relative energies of $d^{\mathrm{n}} s$ and $d^{\mathrm{n}-1} s^{2}$ had been determined for Tc as described above, it was called to my attention that an approximate value of this difference was predicted in 1928 by Gibbs and White [9].

TABLE 4. Spectral terms of $\mathrm{Mn}$ I and $\mathrm{Te} \mathrm{I}$

\begin{tabular}{|c|c|c|c|c|c|c|c|}
\hline \multirow{2}{*}{$\begin{array}{l}\text { Electron } \\
\text { configuration }\end{array}$} & \multirow{2}{*}{$\begin{array}{c}\text { Term } \\
\text { symbol }\end{array}$} & \multirow{2}{*}{$J$} & \multicolumn{2}{|c|}{ Mn I } & \multicolumn{2}{|c|}{$\operatorname{Tc} \mathrm{I}$} & \multirow{2}{*}{$\mathrm{Mn} \mathrm{I}-\mathrm{Te} \mathrm{I}$} \\
\hline & & & Level & Interval & Level & Interval & \\
\hline $3 d^{5} 4 s\left({ }^{7} \mathrm{~S}^{5} \mathrm{~S}\right) 4 s$ & $a{ }^{6} \mathrm{~S}$ & $2 \frac{1}{2}$ & 0.0 & & 0.0 & & 0 \\
\hline $3 d^{6}\left(a^{5} \mathrm{D}\right) 4 s$ & $a^{6} \mathrm{D}$ & $\begin{array}{l}41 / 2 \\
31 / 2 \\
21 / 2 \\
11 / 2 \\
0^{1 / 2}\end{array}$ & $\begin{array}{l}17052.3 \\
17282.0 \\
17451.5 \\
17568.5 \\
17637.2\end{array}$ & $\begin{array}{r}-229.7 \\
-169.5 \\
-117.0 \\
-68.7\end{array}$ & $\begin{array}{l}2572.9 \\
3250.9 \\
3700.6 \\
4002.7 \\
4178.8\end{array}$ & $\begin{array}{l}-678.0 \\
-449.7 \\
-302.1 \\
-176.1\end{array}$ & $\begin{array}{l}14479 \\
14031 \\
13751 \\
13566 \\
13458\end{array}$ \\
\hline $3 d^{5} 4 s\left(a^{7} \mathbf{S}\right) 4 p$ & $z^{8} \mathrm{P}^{\circ}$ & $\begin{array}{l}41 / 2 \\
3^{1 / 2} \\
2^{1 / 2}\end{array}$ & $\begin{array}{l}18705.4 \\
18531 . \% \\
18402.5\end{array}$ & $\begin{array}{l}\text { 173. } 7 \\
129.2\end{array}$ & $\begin{array}{l}17523.0 \\
16874.5 \\
16428.8\end{array}$ & $\begin{array}{l}\text { 648. } 5 \\
445.7\end{array}$ & $\begin{array}{l}1182 \\
1657 \\
1974\end{array}$ \\
\hline $3 d^{6}\left(a^{5} \mathrm{D}\right) 4 s$ & $a^{4} \mathrm{D}$ & $\begin{array}{l}31 / 2 \\
21 / 2 \\
11 / 2 \\
01 / 2\end{array}$ & $\begin{array}{l}23296.7 \\
23549.2 \\
23719.5 \\
23818.8\end{array}$ & $\begin{array}{r}-252.5 \\
-170.3 \\
-99.3\end{array}$ & $\begin{array}{l}10516.5 \\
11063.0 \\
11578.5 \\
11890.7\end{array}$ & $\begin{array}{l}-546.5 \\
-515.5 \\
-312.2\end{array}$ & $\begin{array}{l}12780 \\
12486 \\
12141 \\
11928\end{array}$ \\
\hline $3 d^{5} 4 s\left(a^{7} \mathrm{~S}\right) 4 p$ & $z^{\beta} \mathrm{P}^{\circ}$ & $\begin{array}{l}31 / 2 \\
21 / 2 \\
11 / 2\end{array}$ & $\begin{array}{l}24802.2 \\
24788.0 \\
24779.3\end{array}$ & $\begin{array}{r}\text { 14. } 2 \\
8.7\end{array}$ & $\begin{array}{l}23265.4 \\
23455.3 \\
23588.5\end{array}$ & $\begin{array}{l}-189.9 \\
-133.2\end{array}$ & $\begin{array}{l}1537 \\
1333 \\
1191\end{array}$ \\
\hline
\end{tabular}


TABLE 4. Spectral terms of Mn I and Te I-Continued

\begin{tabular}{|c|c|c|c|c|c|c|c|}
\hline \multirow{2}{*}{$\begin{array}{c}\text { Electron } \\
\text { configuration }\end{array}$} & \multirow{2}{*}{$\begin{array}{l}\text { Term } \\
\text { symbol }\end{array}$} & \multirow{2}{*}{$J$} & \multicolumn{2}{|c|}{ Mn I } & \multicolumn{2}{|c|}{ Te I } & \multirow{2}{*}{$\mathrm{Mn} \mathrm{I}-\mathrm{Te} \mathrm{I}$} \\
\hline & & & Level & Interval & Level & Interval & \\
\hline $3 d^{5} 4 s\left(a^{5} \mathrm{~S}\right) 4 p$ & $z^{4} \mathrm{P}^{\circ}$ & $\begin{array}{l}21 / 2 \\
11 / 2 \\
01 / 2\end{array}$ & $\begin{array}{l}31001.1 \\
31076.4 \\
31124.9\end{array}$ & $\begin{array}{l}-75.3 \\
-48.5\end{array}$ & $\begin{array}{l}28989.4 \\
29441.9 \\
29902.1\end{array}$ & $\begin{array}{l}-452.5 \\
-460.2\end{array}$ & $\begin{array}{l}2012 \\
1634 \\
1223\end{array}$ \\
\hline $3 d^{5} 4 s\left(a{ }^{5} \mathbf{S}\right) 4 p$ & $y{ }^{6} \mathrm{P}^{\circ}$ & $\begin{array}{l}31 / 2 \\
21 / 2 \\
11 / 2\end{array}$ & $\begin{array}{l}35769.9 \\
35725.8 \\
35690.0\end{array}$ & $\begin{array}{l}\text { 44. } 1 \\
35.8\end{array}$ & $\begin{array}{l}3192 \% .0 \\
32258.0\end{array}$ & -331.1 & $\begin{array}{l}3799 \\
3432\end{array}$ \\
\hline $3 d^{5} 4 s\left(a^{7} \mathrm{~S}\right) 5 s$ & $e^{8} \mathrm{~S}$ & $31 / 2$ & 39431. 3 & & 37612.5 & & 1819 \\
\hline $3 d^{5} 4 s\left(a^{7} \mathrm{~S}\right) 5 s$ & $e^{6} \mathrm{~S}$ & $2 \frac{1}{2}$ & 41403. 9 & & 39599.0 & & 1805 \\
\hline $3 d^{6}\left(a^{5} \mathrm{D}\right) 4 p$ & $z^{6} \mathrm{D}^{\circ}$ & $\begin{array}{l}41 / 2 \\
31 / 2 \\
21 / 2 \\
11 / 2 \\
01 / 2\end{array}$ & $\begin{array}{l}41789.4 \\
41932.6 \\
42053.7 \\
42143.5 \\
42198.6\end{array}$ & $\begin{array}{r}-143.2 \\
-121.1 \\
-89.8 \\
-55.1\end{array}$ & $\begin{array}{l}27369.8 \\
27660.1 \\
27940.8 \\
28151.4 \\
28296.7\end{array}$ & $\begin{array}{l}-290.3 \\
-280.7 \\
-210.6 \\
-145.3\end{array}$ & $\begin{array}{l}14420 \\
14272 \\
14113 \\
13992 \\
13902\end{array}$ \\
\hline $3 d^{6}\left(a^{5} \mathrm{D}\right) 4 p$ & $z^{6} \mathrm{~F}^{\circ}$ & $\begin{array}{l}51 / 2 \\
4112 \\
31 / 2 \\
21 / 2 \\
11 / 2 \\
01 / 2\end{array}$ & $\begin{array}{l}43314.2 \\
43428.6 \\
43524.1 \\
43595.5 \\
43644.4 \\
43673.0\end{array}$ & $\begin{array}{r}-114.4 \\
-95.5 \\
-71.4 \\
-48.9 \\
-28.6\end{array}$ & $\begin{array}{l}30067.1 \\
30133.1 \\
30382.0 \\
30528.8 \\
30630.5 \\
30689.1\end{array}$ & $\begin{array}{r}-66.0 \\
-248.9 \\
-146.8 \\
-101.8 \\
-58.6\end{array}$ & $\begin{array}{l}13247 \\
13296 \\
13142 \\
13067 \\
13014 \\
12984\end{array}$ \\
\hline $3 d^{6}\left(a^{5} \mathrm{D}\right) 4 p$ & $z^{4} \mathrm{~F}^{\circ}$ & $\begin{array}{l}41 / 2 \\
31 / 2 \\
21 / 2 \\
11 / 2\end{array}$ & $\begin{array}{l}44288.8 \\
44523.5 \\
44696.3 \\
44814.6\end{array}$ & $\begin{array}{l}-234.7 \\
-172.8 \\
-118.3\end{array}$ & $\begin{array}{l}31114.0 \\
31604.9 \\
32014.8 \\
32253.5\end{array}$ & $\begin{array}{l}-490.9 \\
-409.9 \\
-238.7\end{array}$ & $\begin{array}{l}13175 \\
12919 \\
12682 \\
12561\end{array}$ \\
\hline $3 d^{6}\left(a^{5} \mathrm{D}\right) 4 p$ & $x^{6} \mathrm{P}^{\circ}$ & $\begin{array}{l}31 / 2 \\
21 / 2 \\
11 / 2\end{array}$ & $\begin{array}{l}44993.9 \\
45156.1 \\
45259.2\end{array}$ & $\begin{array}{l}-162.2 \\
-103.1\end{array}$ & $\begin{array}{l}31414.0 \\
31406 . \% \\
31503.8\end{array}$ & $\begin{array}{r}7.3 \\
-97.1\end{array}$ & $\begin{array}{l}13580 \\
13749 \\
13755\end{array}$ \\
\hline $3 d^{6}\left(a^{5} \mathrm{D}\right) 4 p$ & $z^{4} \mathrm{D}^{\circ}$ & $\begin{array}{l}3^{1 / 2} \\
2^{1} / 2 \\
11 / 2 \\
01 / 2\end{array}$ & $\begin{array}{l}45754.2 \\
45940.9 \\
46083.9 \\
46169.9\end{array}$ & $\begin{array}{r}-186.7 \\
-143.0 \\
-86.0\end{array}$ & $\begin{array}{l}92620.3 \\
33055.6 \\
33343.1 \\
33480.2\end{array}$ & $\begin{array}{l}-465.3 \\
-257.5 \\
-137.1\end{array}$ & $\begin{array}{l}13134 \\
12855 \\
12741 \\
12690\end{array}$ \\
\hline $3 d^{5} 4 s\left(a^{7} \mathbf{S}\right) 4 d$ & $e^{8} \mathrm{D}$ & $\begin{array}{l}51 / 2 \\
41 / 2 \\
31 / 2 \\
21 / 2 \\
11 / 2\end{array}$ & $\begin{array}{l}\text { 46712. } 6 \\
46710.2 \\
46708.3 \\
46707.0 \\
46706.1\end{array}$ & $\begin{array}{l}\text { 2. } 4 \\
\text { 1. } 9 \\
\text { 1. } 3 \\
\text { 0. } 9\end{array}$ & $\begin{array}{l}\text { 44365. } 3 \\
44352.5 \\
44343.9 \\
44337.5 \\
44333.1\end{array}$ & $\begin{array}{r}\text { 12. } 8 \\
8.6 \\
6.4 \\
4.4\end{array}$ & $\begin{array}{l}2347 \\
2358 \\
2364 \\
2370 \\
2373\end{array}$ \\
\hline $3 d^{6} 4 s\left(a^{5} \mathrm{D}\right) 4 p$ & $y{ }^{4} \mathrm{P}^{\circ}$ & $\begin{array}{l}21 / 2 \\
11 / 2 \\
01 / 2\end{array}$ & $\begin{array}{l}46901.1 \\
47154.5 \\
47299.3\end{array}$ & $\begin{array}{l}-253.4 \\
-144.8\end{array}$ & $\begin{array}{l}34516.0 \\
34942.4 \\
35276.2\end{array}$ & $\begin{array}{l}-426.4 \\
-333.8\end{array}$ & $\begin{array}{l}12385 \\
12212 \\
12023\end{array}$ \\
\hline $3 d^{5} 4 s\left(a^{7} \mathrm{~S}\right) 5 p$ & $w^{6} \mathrm{P}^{\circ}$ & $\begin{array}{l}31 / 2 \\
21 / 2 \\
11 / 2\end{array}$ & $\begin{array}{l}4738 \% .6 \\
47659.5 \\
47782.4\end{array}$ & $\begin{array}{l}-271.9 \\
-122.9\end{array}$ & $\begin{array}{l}38240.6 \\
38216.6 \\
38319.4\end{array}$ & $\begin{array}{r}24.0 \\
-102.8\end{array}$ & $\begin{array}{l}9147 \\
9443 \\
9463\end{array}$ \\
\hline $3 d^{5} 4 s\left(a^{5} \mathrm{P}\right) 4 p$ & $y^{6} \mathrm{D}^{\circ}$ & $\begin{array}{l}41 / 2 \\
31 / 2 \\
21 / 2 \\
11 / 2 \\
01 / 2\end{array}$ & $\begin{array}{l}47903.8 \\
47774.5 \\
47754.0 \\
47466.7 \\
47452.2\end{array}$ & $\begin{array}{r}129.3 \\
20.5 \\
287.3 \\
14.5\end{array}$ & $\begin{array}{l}3918 \% .8 \\
38766.0 \\
38600.3 \\
39891.0 \\
39776.1\end{array}$ & $\begin{array}{r}416.8 \\
165.7 \\
-1290.7 \\
114.9\end{array}$ & $\begin{array}{l}8719 \\
9009 \\
9154 \\
7576 \\
7676\end{array}$ \\
\hline $3 d^{5} 4 s\left(a^{7} \mathrm{~S}\right) 6 s$ & $f^{8} \mathrm{~S}$ & $31 / 2$ & 50157.6 & & 49573. 3 & & 584 \\
\hline $3 d^{5} 4 s\left(a^{7} \mathrm{~S}\right) 6 s$ & $g^{6} \mathrm{~S}$ & $21 / 2$ & 50904.6 & & 49717. 3 & & 1187 \\
\hline
\end{tabular}


Departures from the Landé interval rule occur in both spectra, but they are occasionally much greater in Te I than in Mn I. For example, the interval between the two largest $J$ values of $z{ }^{6} \mathrm{~F}^{\circ}$ is abnormally small in Te $\mathrm{I}$, and $x^{6} \mathrm{P}^{\circ}$ is only partially inverted in Tc I. Despite careful search the $J=3 \frac{1}{2}$ level of $y^{6} \mathrm{P}^{\circ}$ could not be found. The intervals in $y^{6} \mathrm{D}^{\circ}$ are extremely irregular in both spectra. On the whole, both term intervals and line intensities indicate that, to a large degree, the optical electrons of Tc atoms obey $L S$-coupling. It is planned to test this by observing the Zeeman effect, and incidentally to check the proposed designations of Tc atomic energy levels.

\section{First Ionization Potential of Tc}

If two or more spectral terms form a Rydberg. series, that series may be extrapolated to a limiting wave number that represents the energy of binding of the last bound electron. In complex atoms, such as those under discussion, the number of excited states is relatively large in successive shells, and the transitions from high terms in series either give faint lines or are not observed. However, as long ago as 1894, Kayser and Runge [10] detected the first spectral series in Mn I. That series, now interpreted as $z^{8} \mathrm{P}^{\circ}-n^{8} \mathrm{~S}$, permitted a calculation of the absolute value of $z^{8} \mathrm{P}^{\circ}$, and then by addition of the intersystem combination $a^{6} \mathrm{~S}-z^{8} \mathrm{P}^{\circ}$ the value of the term $a^{6} \mathrm{~S}$ representing the normal state of the atom was arrived at. A similar procedure is now possible with Te I because two multiplets (of three lines each) have been found and interpreted as combinations of $z^{8} \mathrm{P}^{\circ}$ with successive series terms $e^{8 \mathrm{~S}}$ and $f^{8} \mathrm{~S}$. These combinations, $z^{8} \mathrm{P}^{\circ}-n^{8} \mathrm{~S}$, as well as the intersystem lines, $a^{6} \mathrm{~S}-z^{8} \mathrm{P}^{\circ}$, are shown in table 3 . A Rydberg interpolation table applied to the series lines yields the following approximate values for the $z^{8} \mathrm{P}^{\circ}$ levels of $\mathrm{Te} \mathrm{I}$ :

$$
\begin{aligned}
& z^{8} \mathrm{P}_{41 / 2}^{\circ}=42766 \\
& z^{8} \mathrm{P}_{31 / 2}^{\circ}=43415 \\
& z^{8} \mathrm{P}_{21 / 2}^{\circ}=43860
\end{aligned}
$$

Adding the wave numbers of the resonance lines, we obtain the following absolute values for the $a^{6} \mathrm{~S}_{21 / 2}$ term:

$$
\begin{aligned}
a^{6} \mathrm{~S}_{21 / 2} & =z^{8} \mathrm{P}_{31 / 2}^{\circ}+\left(a^{6} \mathrm{~S}_{21 / 2}-z^{8} \mathrm{P}_{31 / 2}^{\circ}\right)=43415+16875 \\
& =60290 \mathrm{~cm}^{-1}, \\
a^{6} \mathrm{~S}_{21 / 2} & =z^{8} \mathrm{P}_{21 / 2}^{\circ}+\left(a^{6} \mathrm{~S}_{21 / 2}-z^{8} \mathrm{P}_{21 / 2}^{\circ}\right)=43860+16429 \\
& =60289 \mathrm{~cm}^{-1} .
\end{aligned}
$$

Assuming that 1 electron volt $=8067.5 \mathrm{~cm}^{-1}$, this value of the deepest term in the Tc I spectrum corresponds to an ionization potential of 7.47 electron volts.

Experience tells us that extrapolation of a twoterm Rydberg series generally leads to a slightly too-large value for the limit, which is more accurateiy given by a Ritz formula of the type $\nu=L-R(n+\mu+$ $\alpha T)^{2}$. In the spectral series for $37 \mathrm{Rb}, 46 \mathrm{Pd}$, and $47 \mathrm{Ag}$ the Ritz correction $\alpha$ has an average value of $2.0 \times 10^{-6}$. Assuming that this correction is valid also for $43 \mathrm{Tc}$, the absolute value of the $a{ }^{6} \mathrm{~S}_{21 / 2}$ term of Tc I is found to be $60070 \mathrm{~cm}^{-1}$ and the corresponding ionization potential is $60070 / 8067.5=7.45$ electron volts.

\section{Second Spectrum of Technetium, TC II}

\section{TC II Multiplets}

Within the wavelength limits $2261.30 \mathrm{~A}$ and $4434.96 \mathrm{~A}$ about 800 spectral lines have been definitely identified as belonging to singly ionized Tc atoms, the Tc II spectrum. The short-wave limit was imposed by the smallness of the Tc sample and by the declining efficiency of photographic plates and spectrograph employed in recording the spark spectrum. The paucity of enhanced Tc II lines in the visible spectrum is responsible for the long-wave limit of observation. The measured wavelengths of Te II lines were converted to vacuum wave numbers with Kayser's [7] Tabelle der Schwingungszahlen.

Among the observed Te II lines there are two ultraviolet multiplets, each of three intense lines, which were immediately recognized as the combinations $a^{7} \mathrm{~S}_{3}-z^{7} \mathrm{P}_{2,3,4}^{\circ}(2647.02,2610.00,2543.24 \mathrm{~A})$ and $a{ }^{5} \mathrm{~S}_{2}-z{ }^{5} \mathrm{P}_{1,2,3}^{\circ}(3195.21,3212.01,3237.02 \mathrm{~A})$. In the Mn II spectrum $a^{7} \mathrm{~S}_{3}$ is the ground state, and $9473 \mathrm{~cm}^{-1}$ lower than $a{ }^{5} \mathrm{~S}_{2}$. Assuming that a similar state of affairs could be expected in the Tc II spectrum, combinations between the quintet and septet terms were sought in order to fix their relative values. Fortunately, these intersystem combinations were unmistakeably represented by two outstanding lines (3892.14 and $3975.02 \mathrm{~A}$ ), at the short-wave edge of

\begin{tabular}{|c|c|c|}
\hline & $\begin{array}{l}a{ }^{7} \mathrm{~S}_{3} \\
0.0\end{array}$ & $\begin{array}{c}a{ }^{5} \mathrm{~S}_{2} \\
12617.1\end{array}$ \\
\hline $\begin{array}{l}z^{7} \mathrm{P}_{4}^{\circ} \\
39308.1 \\
z^{7} \mathrm{P}_{3}^{\circ} \\
38302.7 \\
z^{7} \mathrm{P}_{2}^{\circ} \\
37767.1\end{array}$ & $\begin{array}{l}\text { 2543. } 24(1000) \\
39308.1 \\
2610.00(800) \\
38302.7 \\
2647.02(600) \\
37767.1\end{array}$ & $\begin{array}{l}3892.14(40 c) \\
25685.6 \\
3975.02(10) \\
25150.0\end{array}$ \\
\hline $\begin{array}{l}z^{5} \mathrm{P}_{3}^{\circ} \\
43500.8 \\
z^{5} \mathrm{P}_{2}^{\circ} \\
43741.3 \\
z^{5} \mathrm{P}_{1}^{\circ} \\
43904.9\end{array}$ & $\begin{array}{l}2298.10(10) \\
43500.8 \\
2285.47(3) \\
43741.2\end{array}$ & $\begin{array}{l}3237.02(500 c) \\
30883.7 \\
3212.01(300 c) \\
31124.2 \\
\text { 3195. } 21(200 c) \\
\text { 31287. } 8\end{array}$ \\
\hline
\end{tabular}
the visible spectrum, and by the two most prominent lines (2298.10 and $2285.47 \mathrm{~A}$ ) very near the ultraviolet limit of observation. These Tc in multiplets are presented in table 5, which is constructed like tables $1,2,3$. The Tc II terms revealed by these

TABLE 5. Multiplets in the Te II spectrum 
TABLE 6. Spectral terms of Mn II and Te II

\begin{tabular}{|c|c|c|c|c|c|c|c|}
\hline \multirow{2}{*}{$\begin{array}{c}\text { Electron } \\
\text { configuration }\end{array}$} & \multirow{2}{*}{$\begin{array}{c}\text { Term } \\
\text { symbol }\end{array}$} & \multirow{2}{*}{$J$} & \multicolumn{2}{|c|}{ Mn II } & \multicolumn{2}{|l|}{ Te II } & \multirow{2}{*}{$\mathrm{Mn} \mathrm{II}-\mathrm{Te} \mathrm{II}$} \\
\hline & & & Level & Interval & Level & Interval & \\
\hline $3 d^{5}\left(a^{6} \mathbf{S}\right) 4 s$ & $a^{7} \mathrm{~S}$ & 3 & 0.0 & & $0.0+x$ & & $x$ \\
\hline $3 d^{5}\left(a^{6} \mathbf{S}\right) 4 s$ & $a^{5} \mathrm{~S}$ & 2 & 9472.9 & & 12617. $1+x$ & & $-3244+x$ \\
\hline $3 d^{5}\left(a^{6} \mathrm{~S}\right) 4 p$ & $z^{7} \mathrm{P}^{\circ}$ & $\begin{array}{l}4 \\
3 \\
2\end{array}$ & $\begin{array}{l}38806.5 \\
38543.0 \\
38366.1\end{array}$ & $\begin{array}{l}263.5 \\
176.9\end{array}$ & $\begin{array}{l}\text { 39308. } 1+x \\
\text { 38302. } 1+x \\
\text { 37\%6\%. } 1+x\end{array}$ & $\begin{array}{r}1005.4 \\
535.6\end{array}$ & $\begin{array}{r}-502+x \\
+240+x \\
599+x\end{array}$ \\
\hline $3 d^{5}\left(a{ }^{6} \mathbf{S}\right) 4 p$ & $z^{5} \mathrm{P}^{\circ}$ & $\begin{array}{l}3 \\
2 \\
1\end{array}$ & $\begin{array}{l}43370.4 \\
43484.5 \\
4355 \% .0\end{array}$ & $\begin{array}{r}-114.1 \\
-72.5\end{array}$ & $\begin{array}{l}43500.8+x \\
43741.3+x \\
43904.9+x\end{array}$ & $\begin{array}{l}-240.5 \\
-163.5\end{array}$ & $\begin{array}{l}-130+x \\
-257+x \\
-348+x\end{array}$ \\
\hline
\end{tabular}

multiplets are displayed in table 6 alongside the analogous terms in the Mn II spectrum [8]. Attention is called to the large separation $\left(12,617 \mathrm{~cm}^{-1}\right)$ between $a^{7} \mathrm{~S}_{3}$ and $a{ }^{5} \mathrm{~S}_{2}$, and to the fact that the intervals in the $z{ }^{5} \mathrm{P}^{\circ}$ term are almost exactly in the ratio 3 to 2 as required by the Landé interval rule.

In the Mn II spectrum the second metastable state is $3 d^{6} a{ }^{5} \mathrm{D}$, which combines with $3 d^{5} 4 p z^{5} \mathrm{P}^{\circ}$ to produce a prominent multiplet in the ultraviolet (3441.98 to $3497.53 \mathrm{~A}$ ). The analogous multiplet of Te II lines was sought with the aid of the $z{ }^{5} \mathrm{P}^{0}$ intervals, 240.5 and $163.6 \mathrm{~cm}^{-1}$. In view of the stronger binding of $d$-type electrons in the Tc I spectrum, this Te II multiplet must be expected to lie farther in the ultraviolet. Failure to find it among the observed lines forced the conclusion that in Tc II the term $a^{5} \mathrm{D}$ has lower energy than $a^{7} \mathrm{~S}$, and that the multiplet under discussion will therefore have wavelengths outside the present limit of observation of Te spectra. Until this multiplet is observed it is impossible to give the correct values of Tc II terms in table 6 relative to the normal state $a^{5} \mathrm{D}=0.0$; this uncertainty is expressed by $x$. Further progress in the analysis and interpretation of the second spectrum of technetium would be greatly facilitated by extending the observations to both shorter and longer waves than the present limits.

\section{References}

[1] M. A. Catalán, Phil. Trans. Roy. Soc. London 223 A. 127 (1922).

[2] W. Noddack and I. Tacke, Naturwissenschaften 13, 567 (1925).

[3] C. Perrier and E. Segré, J. Chem. Phys. 5, 712 (1937); E. E. Motta, G. E. Boyd, and Q. V. Larson, Phys. Rev. 79, 1270 L (1947).

[4] G. W. Parker, J. Reed, and J. W. Ruch, CNI-1, Clinton National Laboratory, Contract No. W-35-058-eng-71 (Jan. 9, 1948).

[5] C. Pelrier and E. Segré, Nature 159, 24 (1947).

[6] W. F. Meggers and B. F. Scribner, J. Research NBS 45, 476 (1950) RP2161.

[7] H. Kayser, Tabelle der Schwingungszahlen, 1944 ed. (Edwards Brothers, Inc., Ann Arbor, Mich.)

[8] C. E. Moore, Atomic energy levels, NBS Circular 467, Vol. II, in press (1951).

[9] R. C. Gibbs and H. E. White, Proc. Nat. Acad. Sci. 14, 559 (1928).

[10] H. Kayser and C. Runge, Wiedem. Ann. 52, 93 (1894).

Washington, December 17, 1950. 